\title{
Episodio de precipitaciones localmente intensas en La Palma durante el 7 de noviembre de 2017
}

\author{
David Suárez Molina1(dsuarezm@aemet.es) \\ Cristo Alejo Herrera ${ }^{1}$ (calejoh@aemet.es) \\ Javier de Luis Cuartero' ${ }^{1}$ (jluisc@aemet.es)
}

${ }^{1}$ AEMET / Delegación Territorial en Canarias

\begin{abstract}
RESUMEN
El archipiélago canario se encuentra en una región de gran interés desde una perspectiva meteorológica. Situado en el Atlántico Norte suroriental, entre la zona templada y la región subtropical del planeta, Canarias ha sido afectada durante los últimos años por estructuras meteorológicas de variada naturaleza. Entre ellas cabe destacar las bajas atlánticas, depresiones aisladas de niveles altos, frentes activos, estructuras de naturaleza tropical, etc.

Las islas son muy montañosas y cuentan con una compleja orografía, con una altitud media de 415 metros y elevados gradientes altitudinales en algunos casos, ya que se puede pasar del nivel del mar a los 3718 metros en el caso del Teide. Esta compleja orografía es un factor fundamental como mecanismo de disparo en situaciones de inestabilidad atmosférica. Además es una causa eficiente de realce de las precipitaciones.

En este trabajo se analiza el episodio de precipitaciones intensas que afectó al municipio de Garafía (situado en el noroeste de la isla de La Palma) el pasado 7 de noviembre de 2017.
\end{abstract}

PALABRAS CLAVE: predicción numérica; convección; FMA; orografía compleja; precipitaciones torrenciales; alta resolución.

\section{INTRODUCCIÓN}

Durante los pasados días del 7 al 9 de noviembre de 2017 el archipiélago canario estuvo afectado por la presencia en niveles medios y altos de la troposfera, de una depresión aislada de niveles altos (dana) con unas temperaturas en el nivel de $500 \mathrm{hPa}$ entre $-12 \mathrm{y}-16^{\circ} \mathrm{C}$, situada al oeste-suroeste de las islas. En superficie sin embargo, las islas Canarias quedaban bajo la influencia de un potente anticiclón atlántico de unos $1034 \mathrm{hPa}$ situado al oeste de la península ibérica, que generaba un flujo de entre 15 a 25 nudos en superficie sobre las islas.

En una situación general poco significativa destacan dos estructuras mesoescalares con registros sorprendentes. Las precipitaciones de Garafía, donde se registraron $290 \mathrm{~mm}$ en 24 horas y la banda nubosa que dio lugar a abundante aparato eléctrico en el nordeste de Tenerife. Hay que destacar la intensidad de las precipitaciones ya que, del total, $246 \mathrm{~mm}$ cayeron distribuidos entre las 7 y 14 horas.

Este tipo de episodios no debe sorprender, ya que el otoño en Canarias ha dejado en varias ocasiones fenómenos de relevancia y es en general una estación en la que los registros son altos. 
En los siguientes apartados de este documento se realizará un estudio detallado de las principales salidas de modelos numéricos de predicción y herramientas analizadas por los predictores de servicio durante los días previos y el mismo día del evento de precipitaciones intensas que se produjeron en el noroeste de La Palma. En este trabajo se pone énfasis en el día 7, ya que fue el día en que las precipitaciones fueron más intensas, y que por su singularidad merece ser analizado individualmente.

\section{ESTUDIO SINÓPTICO}

Sinópticamente, el pasado 7 de noviembre de 2017, el archipiélago canario quedaba bajo la influencia de un potente anticiclón atlántico de unos $1034 \mathrm{hPa}$ situado al norte de Azores y al oeste de la península ibérica. Por otro lado, había presente una baja sobre el continente africano, al sureste de las islas. Por lo tanto, en superficie Canarias estaba afectada por flujo del nordeste marítimo asociado a las altas presiones de Azores y la parte más oriental del archipiélago se vería influenciada por la entrada de una masa continental africana seca, asociada a la posición de la baja africana.

La situación sinóptica descrita anteriormente se puede observar en la figura 1. En esta figura se representa la presión a nivel del mar en $\mathrm{hPa}$ (con contornos de 4 en 4 unidades de hPa) y la nubosidad compuesta, en la que la nubosidad baja viene representada por color verde, las nubes medias por color azul y las nubes altas por colores rosa.

Se ve que sobre Canarias se extiende el anticiclón con una presión en superficie en torno a 1016 hPa con presencia de nubosidad mixta (mezcla de colores) baja, media y alta.

Además de los centros de presión señalados anteriormente, cabe remarcar la presencia de una estructura en forma de «V» invertida en niveles bajos. Esta onda permitió un flujo del nordeste continuo sobre La Palma. Además el movimiento de dicha estructura hace que las líneas nubosas asociadas al flujo superficial (NE) giren hacia el norte, es decir pasan de tener una componente este dominante, a una componente norte dominante. El giro puede ser importante, para que las líneas nubosas apunten a la zona de interés y no a otras.

Otro aspecto a destacar es el paso de un vórtice en niveles medios de la troposfera justo sobre La Palma, que aparte de aportar inestabilidad térmica, generó una zona de cizalladura direccional vertical y horizontal. Hay que tener en cuenta este factor que en caso de dispararse la convección, esta suele ser estacionaria (mientras el vórtice esté encima) y de gran desarrollo vertical (poca mezcla con el entorno).

En la figura 2 se muestra el entorno sinóptico en el nivel de $500 \mathrm{hPa}$ con las temperaturas sombreadas y su escala a la derecha $\left(\mathrm{en}^{\circ} \mathrm{C}\right)$. Vemos que al oeste-suroeste del archipiélago se situaba un núcleo entre -14 y $-16^{\circ} \mathrm{C}$, ligeramente más cálido que su entorno.

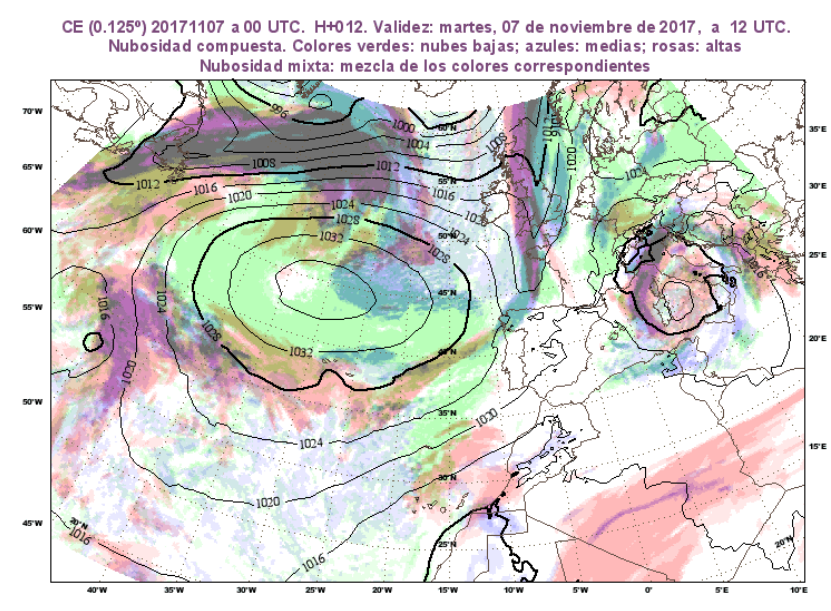

Figura 1. Mapa sinóptico de superficie, del modelo HRES-IFS válido para el 7 de noviembre de 2017 a las 12 UTC.

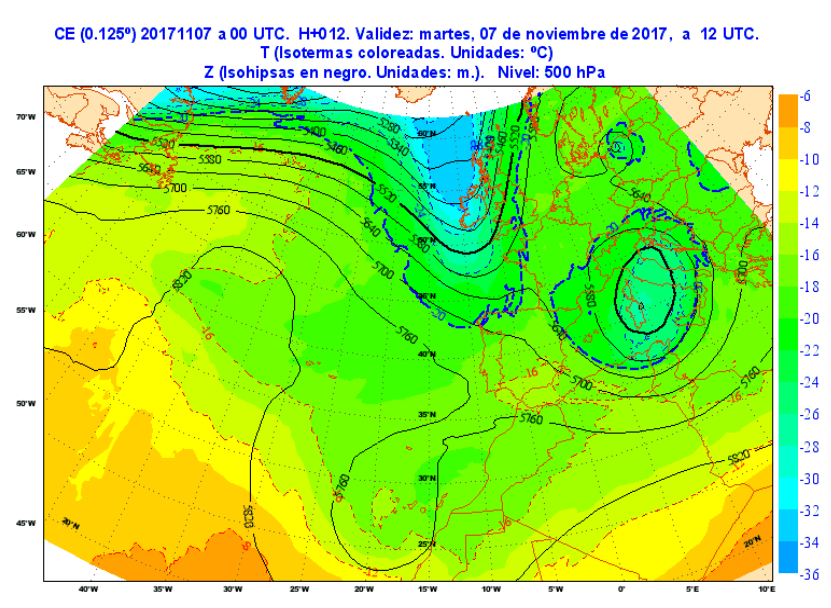

Figura 2. Mapa sinóptico del nivel de 500 hPa, del modelo HRES-IFS válido para el 7 de noviembre de 2017 a las 12 UTC. 
Para terminar con el análisis sinóptico, en las figuras 3 y 4 queda patente el forzamiento dinámico al que quedaba expuesto el archipiélago canario.

Existía en el área de interés un máximo de vorticidad potencial. Las anomalías de vorticidad potencial se conservan en superficies isentrópicas y esta propiedad hace que sea muy útil como herramienta de identificación y evolución de las perturbaciones meteorológicas (Holton, 2004). Este máximo de vorticidad potencial se desplazó en dirección NW-SE sobre las islas, generando ascensos en la parte delantera del máximo de vorticidad ciclónica. Por otro lado, Canarias quedaba en el lado frío del chorro subtropical en la región de salida izquierda. En la región de salida las parcelas de aire deceleran y predominan las divergencias en altura en la zona izquierda (LEHKONEN, 2013). Estas divergencias en altura están relacionadas con convergencias en niveles bajos que producen movimientos ascendentes y en esas áreas pueden producirse precipitaciones intensas.

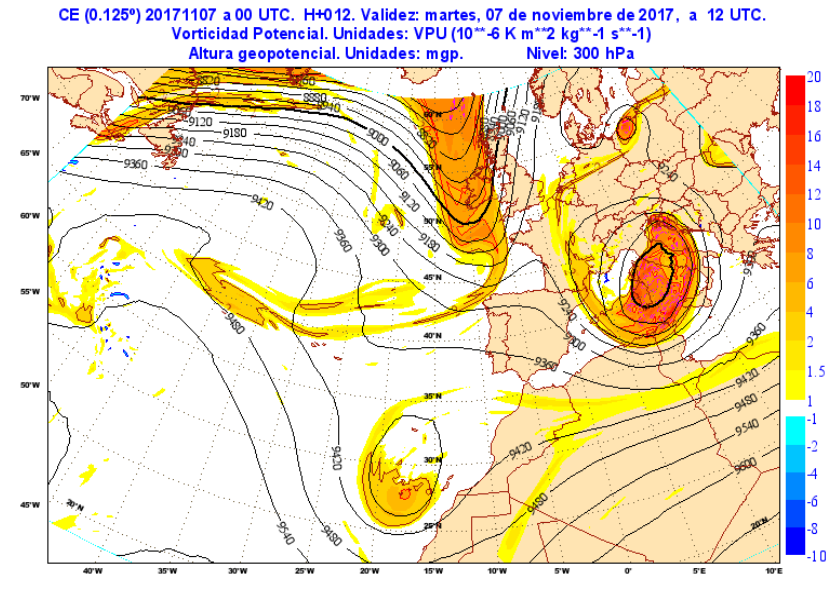

Figura 3. Vorticidad potencial (VPU) y altura geopotencial (mgp) en el nivel de $300 \mathrm{hPa}$, del modelo HRES-IFS válido para el

7 de noviembre de 2017 a las 12 UTC.

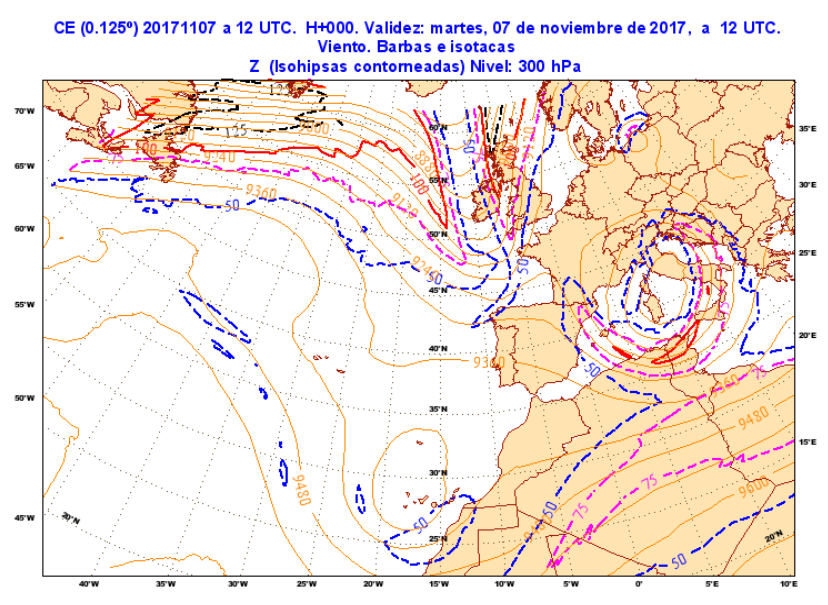

Figura 4. Barbas de viento e isotacas en el nivel de $300 \mathrm{hPa}$, del modelo HRES-IFS válido para el

7 de noviembre de 2017 a las 12 UTC.

\section{ANÁLISIS MESOESCALAR}

\subsection{Masas de aire}

En las proximidades de las islas se pueden identificar dos masas de aire bien diferenciadas, tal y como se ve en las figuras 5 y 6 , donde se muestra el viento y la humedad relativa en $850 \mathrm{hPa}$. Por un lado, la vertiente más oriental del archipiélago, quedaba bajo la influencia de la masa de aire tropical continental (aire más cálido y seco), mientras que la zona más occidental estaría afectada por la masa de aire marítima (aire más frío y húmedo).

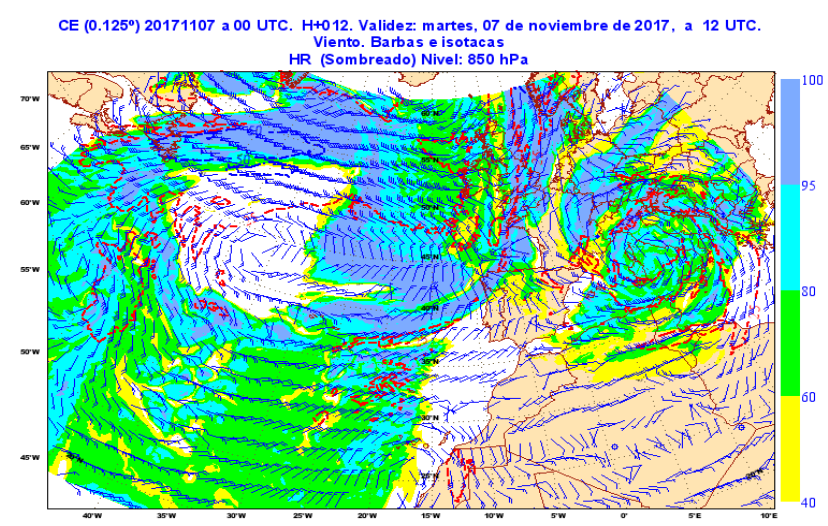

Figura 5. Barbas de viento e isotacas y humedad relativa en el nivel de $850 \mathrm{hPa}$, del modelo

HRES-IFS válido para el 7 de noviembre de 2017 a las 12 UTC (área sinóptica).

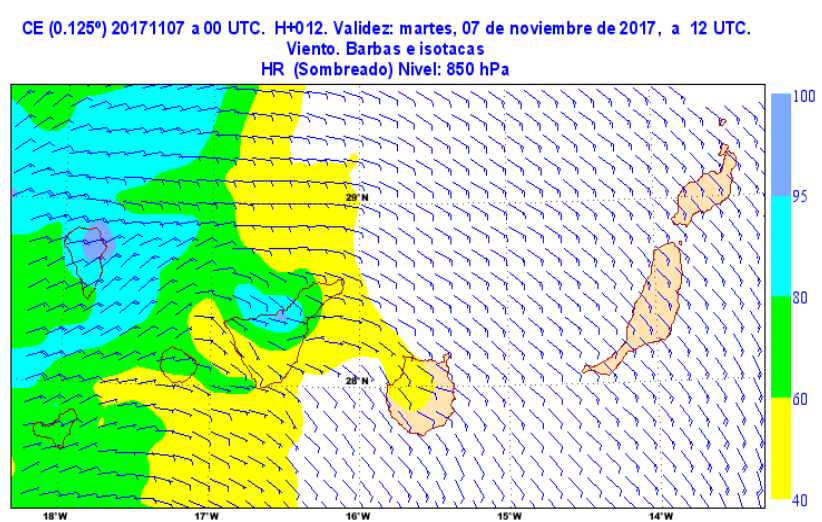

Figura 6. Barbas de viento e isotacas y humedad relativa en el nivel de $850 \mathrm{hPa}$, del modelo HRES-IFS válido para el 7 de noviembre de 2017 a las 12 UTC (área Canarias). 


\subsection{Vientos}

En la figura 7 se muestra el viento a distintos niveles para la isla de La Palma. Se observa que en los niveles de 10 metros, $925 \mathrm{hPa}$ y $850 \mathrm{hPa}$ (figura $7 \mathrm{a}, \mathrm{b}$ y c) predomina un flujo del nordeste con una intensidad de unos 10 a 30 nudos. Queda patente en esta imagen la marcada cizalladura tanto horizontal como vertical, y el aceleramiento del flujo en los extremos noroeste y sureste de la isla, debido al efecto topográfico. La marcada cizalladura pudo aportar una componente de organización a la célula de precipitación que quedaría anclada con la orografía. Este flujo marítimo aportó un gran contenido de humedad en las capas más bajas de la troposfera. Debido a la formación de una mesobaja a sotavento, el viento en la vertiente oeste de la isla fue de componente sur y oeste durante varias horas de la mañana del día 7 de noviembre de 2017. Esta circulación junto con la asociada al alisio (flujo del nordeste) convergía en el extremo noroeste de la isla, pudiendo ser este un mecanismo de disparo de la convección en la zona de interés (Garafía) junto con la variación de dirección de las líneas nubosas debido a la «V» invertida.
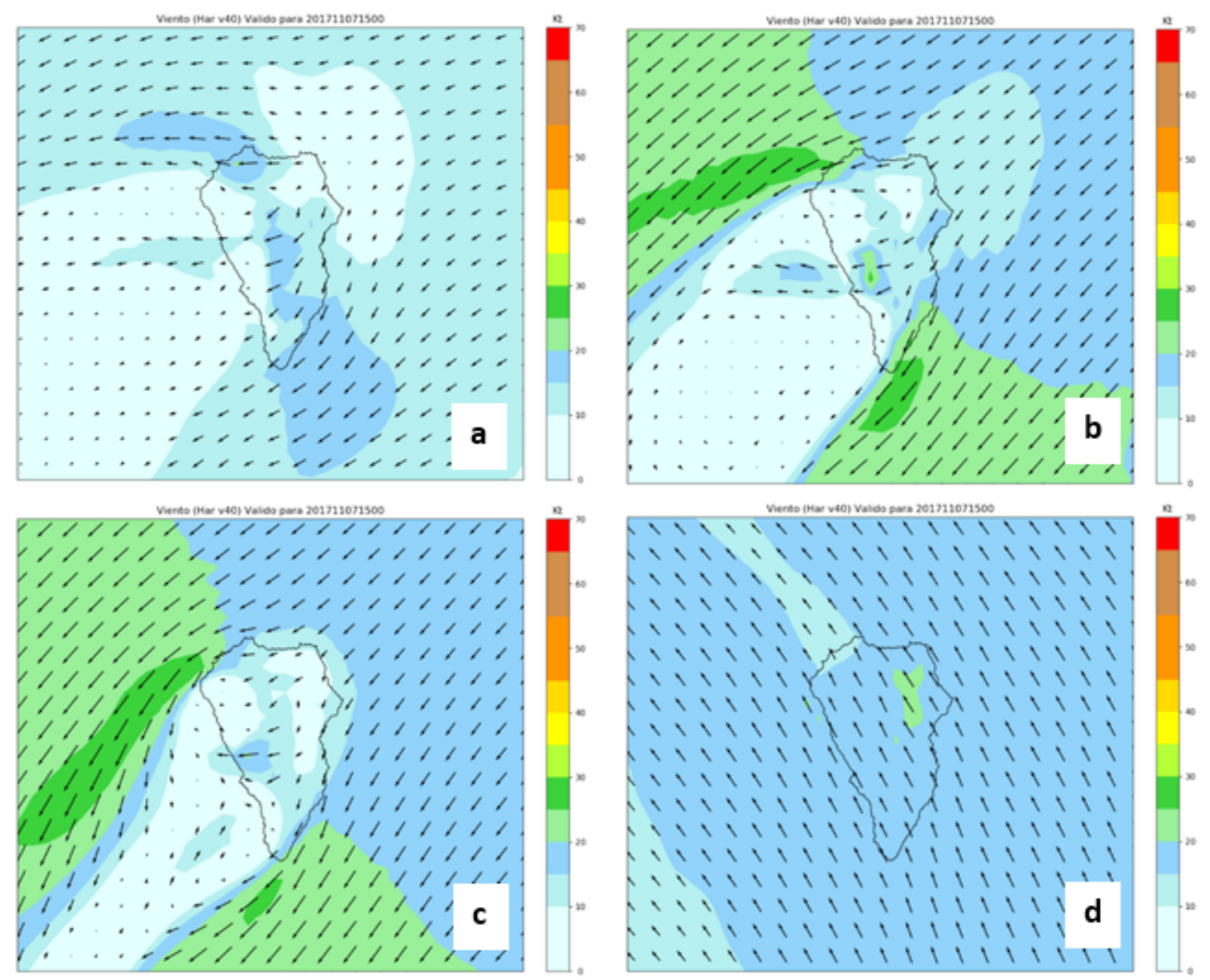

Figura 7. Viento dirección (flechas negras) e intensidad (sombreado en colores en kt), del modelo Harmonie-Arome válido para el 7 de noviembre de 2017 a las 15 UTC.

a) Viento a $10 \mathrm{~m}$, b) $925 \mathrm{hPa}, \mathrm{c}) 850 \mathrm{hPa}$ y d) $700 \mathrm{hPa}$.

\subsection{Mesobaja a sotavento}

Debido a la incidencia del viento en las montañas de la isla y a la acumulación de masa en la cara de barlovento (vertiente este), por efecto del relieve se produce una anomalía de presión positiva a barlovento (mesoalta) y una anomalía de presión negativa a sotavento (mesobaja). 
El efecto de la mesobaja (como se ha comentado en el punto anterior) fue generar vientos de componente marítima y producir una zona de convergencias mesoescalares en el extremo noroeste de La Palma.

Además pudo tener incidencia directa en la disipación de la célula de precipitación, ya que la estructura convectiva se disipó cuando esta mesobaja se debilitó y se produjo un cambio de viento (figura 8) en la vertiente oeste de la isla (el viento giró del segundo y tercer cuadrante a una componente norte), que produjo un descenso de aporte de la humedad que provenía del flujo marítimo.

Como se observa en la figura 8 el giro del viento acaeció en torno a las 12 UTC y la célula de precipitación se disipó alrededor de esa hora.

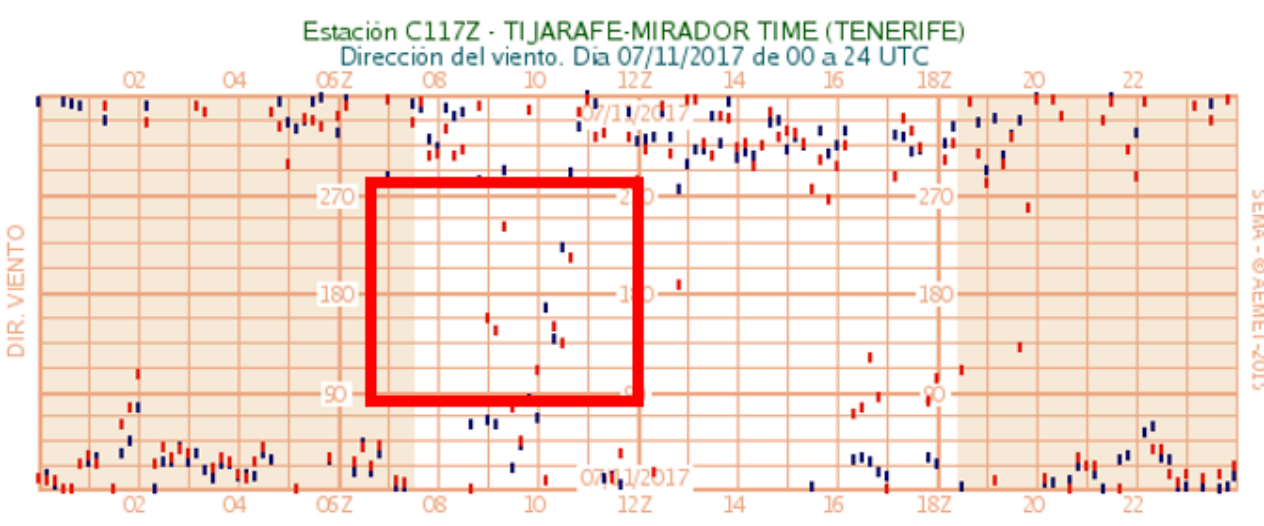

Figura 8.

Gráficos de dirección del viento proveniente de la estación meteorológica automática (ema) de Tijarafe, de la red de estaciones de AEMET, situada al oeste de La Palma.

\subsection{Sondeos}

En la siguiente figura (figura 9), se muestra la estructura vertical de la atmósfera a partir de un sondeo previsto por el modelo HRES-IFS situado al noroeste de la isla de La Palma. Hay que remarcar que según el sondeo previsto, la atmósfera estaría saturada en los primeros 1800 metros. Lo más destacable de los perfiles térmicos en capas bajas, es la presencia de una capa desde superficie hasta prácticamente los $600 \mathrm{hPa}$ con inestabilidad potencial (el gradiente de la temperatura del termómetro húmedo es mayor que el gradiente pseudoadiabático). Además existe un desecamiento de la atmósfera por encima de esta capa inestable. Son de sobra conocidas las subversiones del alisio, debido a capas con inestabilidad potencial (Morán, 1984), que en condiciones normales no provocan precipitaciones importantes ni convección, debido a que la capa adyacente que se sitúa por encima de la inversión del alisio es una capa seca y muy estable. Sin embargo, en este caso la situación es diferente, ya que la presencia de la dana y del máximo de vorticidad al sur de la isla, aportaron la inestabilidad suficiente para que se produjera la subversión.

Figura 9.

Sondeo previsto para las

9 UTC del día 7 de noviembre de 2017, extraído del modelo HRES-IFS de un punto situado al noroeste de

La Palma, de la pasada de

0 UTC del mismo día.
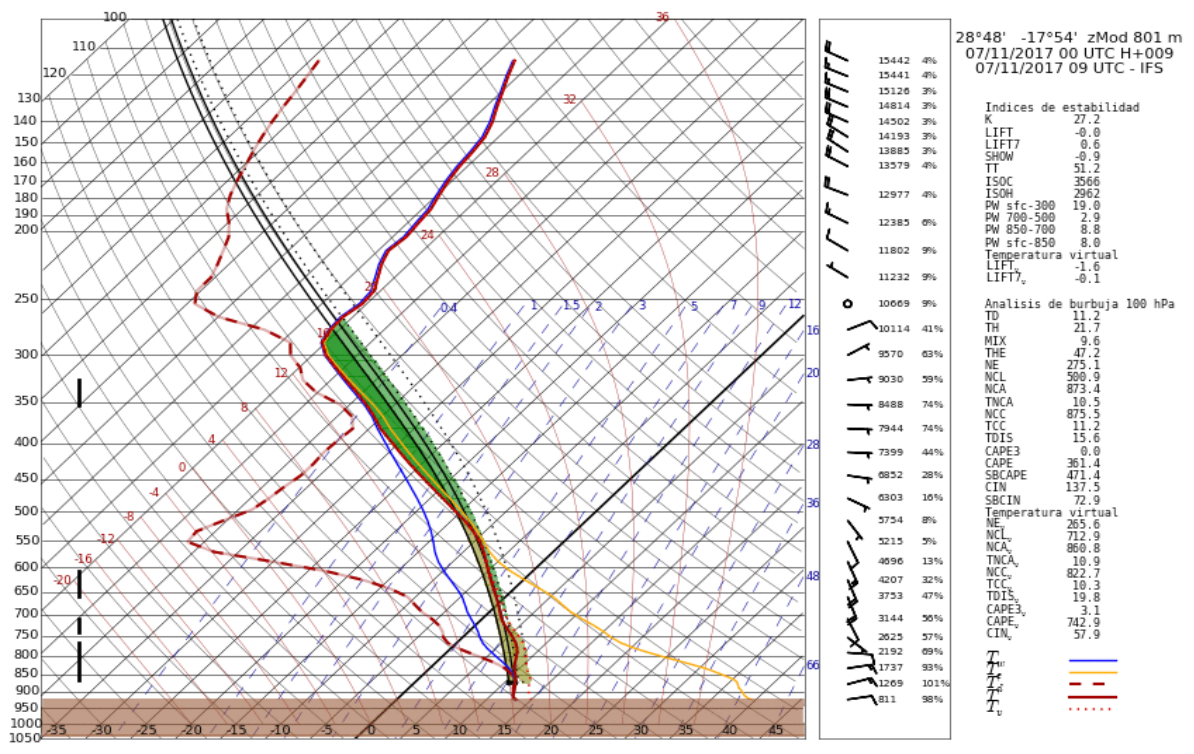
En la figura anterior se expone el perfil vertical de vientos para esta zona de La Palma. El perfil muestra una marcada cizalladura vertical que pudo aportar organización a la estructura.

El CAPE y el SBCAPE previstos por el sondeo fue de 361,4 y 471,4 J/kg respectivamente. Debido a los altos valores de CIN de 137,5 unidades y de lo repartido que estaba el CAPE en la vertical, hizo pensar que el inicio de la convección se vería inhibido y en caso de producirse sería con una baja probabilidad.

\section{COMPORTAMIENTO DE LOS MODELOS}

La variable precipitación es la que sin lugar a dudas genera mayor dificultad a la hora de realizar una predicción, no solo en el contexto cuantitativo sino también en la localización de esta.

Los modelos numéricos de predicción (MNP), muestran limitaciones significativas en nuestra área de interés, en los campos de precipitaciones previstas. Si a esto le sumamos que la precipitación sea de carácter convectivo la incertidumbre aumenta, con lo que es fundamental en un área como Canarias, una región marítima y con una orografía tan compleja, la figura del predictor y las tareas de nowcasting, para poder tomar decisiones y anticiparse a este tipo de situaciones tan locales e impredecibles por los MNP.

En la figura 10 se exponen las salidas previstas de precipitación acumuladas en 24 horas de los principales modelos operativos.

Los modelos deterministas, HRES-IFS (figura 10 a) y Harmonie-Arome (figura 10 b) coincidieron en la isla de La Palma tanto cuantitativamente (entre 15 a 20 mm/24 h) como en la localización de este máximo (en el nordeste de La Palma), y que los modelos coincidan es siempre una buena noticia para los predictores, aunque en numerosas ocasiones, como sucedió este día, ambos modelos fallaran.
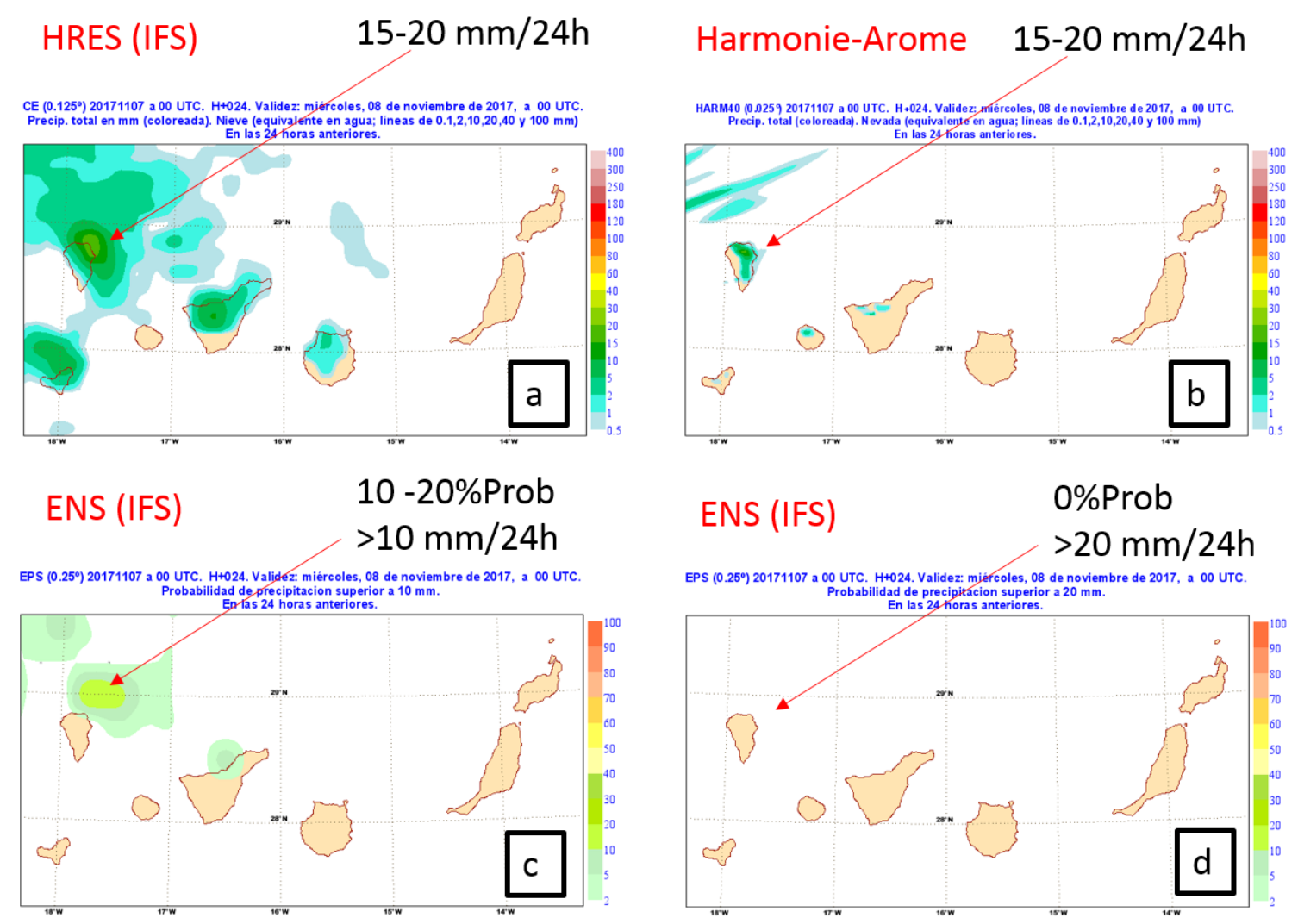

Figura 10. Salidas de precipitación acumulada en 24 horas previstas por los principales modelos numéricos operativos. 
El modelo probabilista (ENS-IFS), por su parte, daba una señal de que las precipitaciones podrían ser superiores a $10 \mathrm{~mm} / 24$ horas con una probabilidad de 10 a $20 \%$ (figura 10 c). Sin embargo, no daba señal de precipitaciones superiores a $20 \mathrm{~mm} / 24$ horas (figura $10 \mathrm{~d}$ ).

En la figura anterior queda constatado que el máximo de precipitación prevista por el modelo no fue bien localizado y evidentemente fue infraestimado.

\section{AVISOS Y PREDICCIONES EMITIDAS}

Debido a la alta incertidumbre que mostraban los modelos numéricos de predicción los días previos, desde el GPV (Grupo de Predicción y Vigilancia) de Canarias, se optó por incluir en las predicciones (emitidas los días previos, D-2 y D-1) autonómica y provincial para la isla de La Palma y zona norte de Tenerife que, con una probabilidad del $30 \%$, las precipitaciones podrían ser localmente fuertes.

En torno a las 7 u 8 UTC del día 7 de noviembre de 2017, como resultado del análisis de las principales herramientas de nowcasting, se decidió avisar telefónicamente a protección civil 112 y se advierte que hay una célula convectiva, al noroeste de la isla precipitando y anclada al relieve y se avisa de que las precipitaciones pueden ser de carácter fuerte.

A partir de los primeros datos recogidos por estaciones meteorológicas externas a la red de estaciones de AEMET, ya que no hay estaciones automáticas de AEMET en la zona donde se produjeron las precipitaciones, se decide emitir un aviso naranja observado por precipitaciones de $50 \mathrm{~mm} / 1$ hora a las 10:28 UTC con finalización a las 15 UTC.

\section{OBSERVACIONES}

Las intensas lluvias en Garafía provocaron inundaciones y la suspensión de clases, hubo servicios públicos, como el suministro eléctrico, afectados aunque no se produjo ningún incidente grave.

En este municipio del noroeste de La Palma un colaborador de AEMET midió 290 mmen 24 horas, aunque del total $246 \mathrm{~mm}$ cayeron distribuidos entre las 7 y 14 horas.

En la figura 11 se muestran las precipitaciones recogidas por las emas de AEMET. A partir de los datos se extrae como conclusión la alta complejidad de la situación debido a lo localizado que fue registrado el máxi-

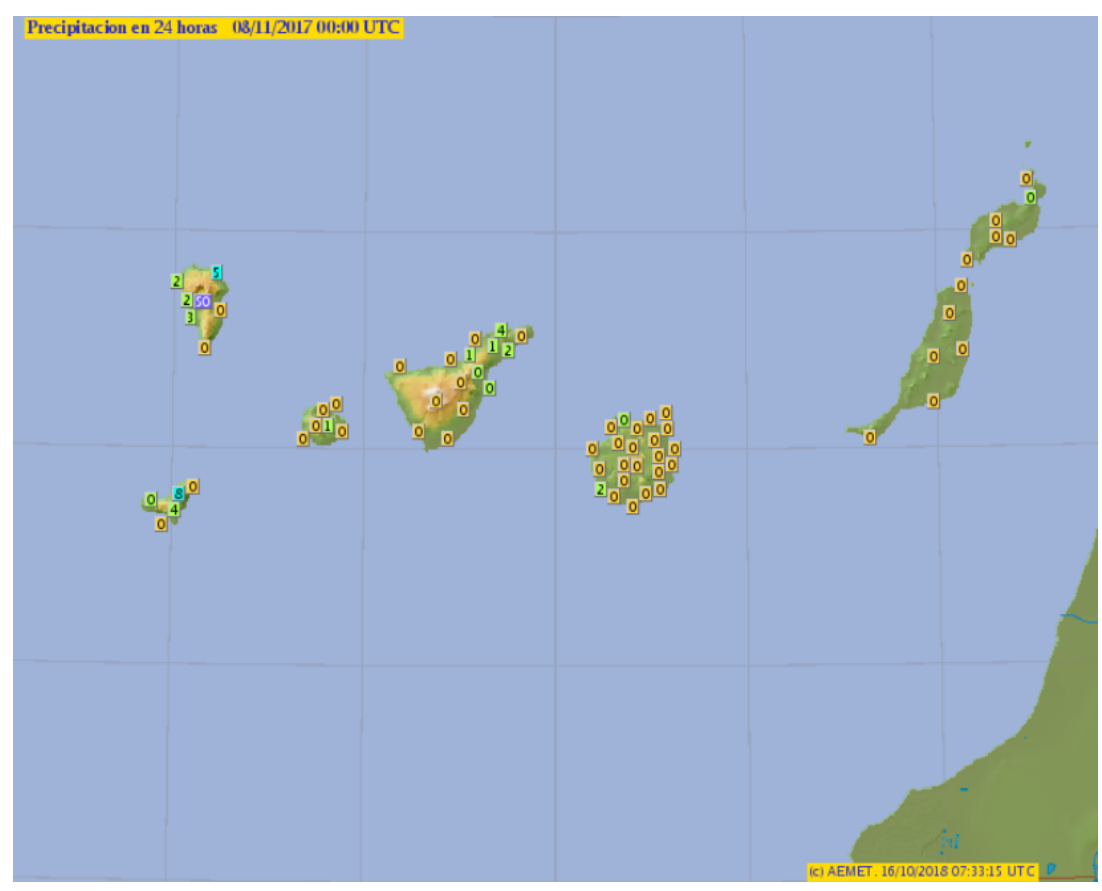
mo de precipitación y podemos observar cómo en las estaciones más cercanas de AEMET a la zona de Garafía, se recogieron entre 2 y $5 \mathrm{~mm} / 24$ horas. En la zona de cumbre, en Mazo (situada a $844 \mathrm{~m}$ ), se registraron $50 \mathrm{~mm}$ a lo largo del día analizado. 
Además de los datos anteriores, destaca un dato de $233 \mathrm{~mm}$ registrado en el noroeste de la isla (acumulado entre las 00 y 14 UTC del día 7 de noviembre de 2017) extraído de la web del Cabildo de La Palma.

En la figura 12 se muestra la imagen PPI observada desde el radar de Las Palmas y las descargas relacionadas con la banda nubosa que dio lugar a abundante aparato eléctrico en el nordeste de Tenerife. Es posible distinguir varios núcleos con reflectividades moderadas entre 38 a 44 dBz. La estructura se desplazó desde el suroeste hacia el nordeste, es decir desde Tenerife hacia el océano, y el paso de esta estructura por la capital tinerfeña dejo un chubasco moderado, en torno a $2 \mathrm{~mm} / \mathrm{h}$.

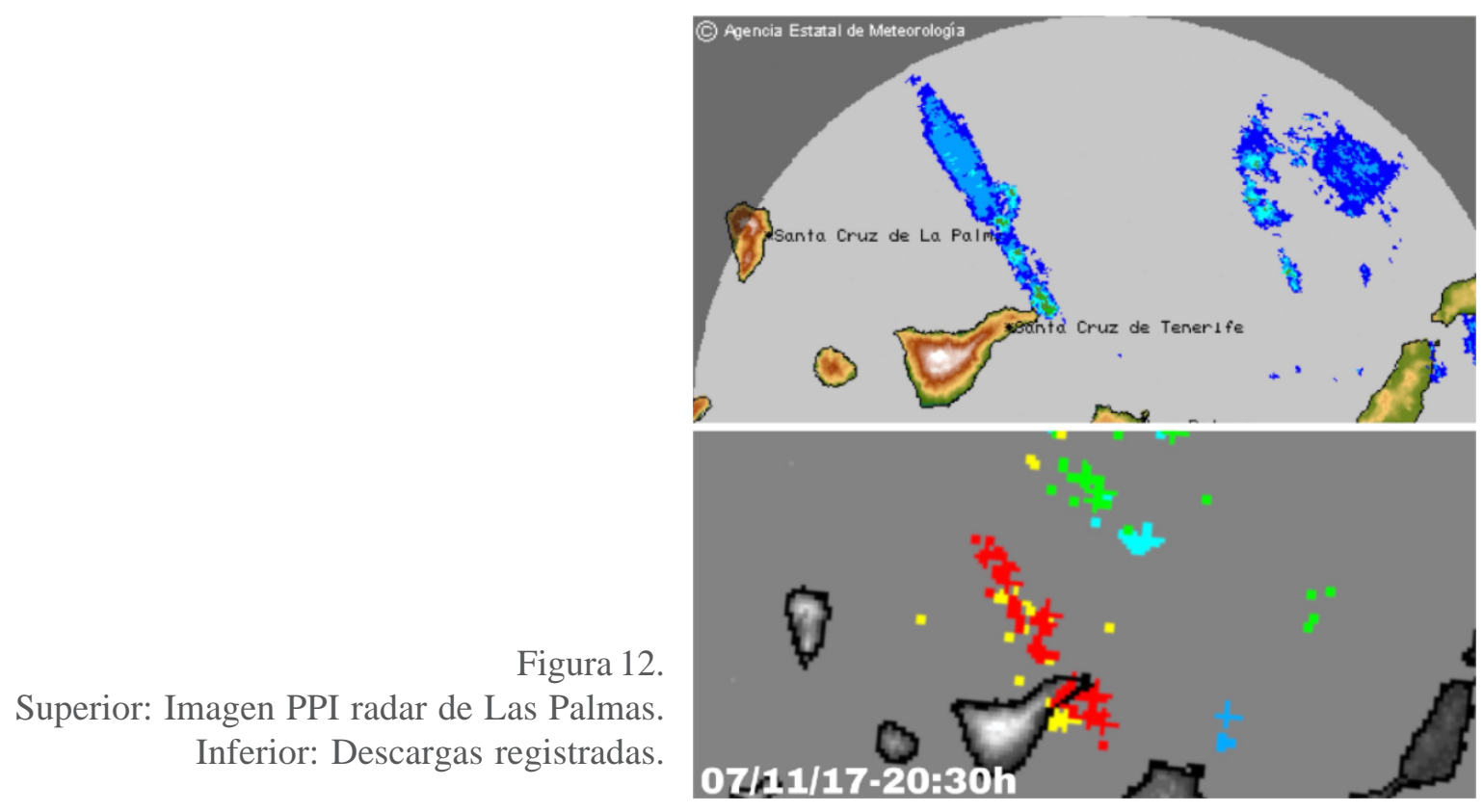

En las siguientes figuras (13 y 14), se observa la célula de precipitación en la imagen del producto sándwich que combina el canal IR10,8 realzado + HRVIS y la imagen del IR10,8 con el realce PREVIMET.

Se observa la célula convectiva situada en el noroeste de La Palma, donde quedaría anclada hasta la fase de disipación, en torno a las 11:15 UTC. El ciclo de vida de esta tormenta unicelular fue del orden de 4 a 5 horas aproximadamente.

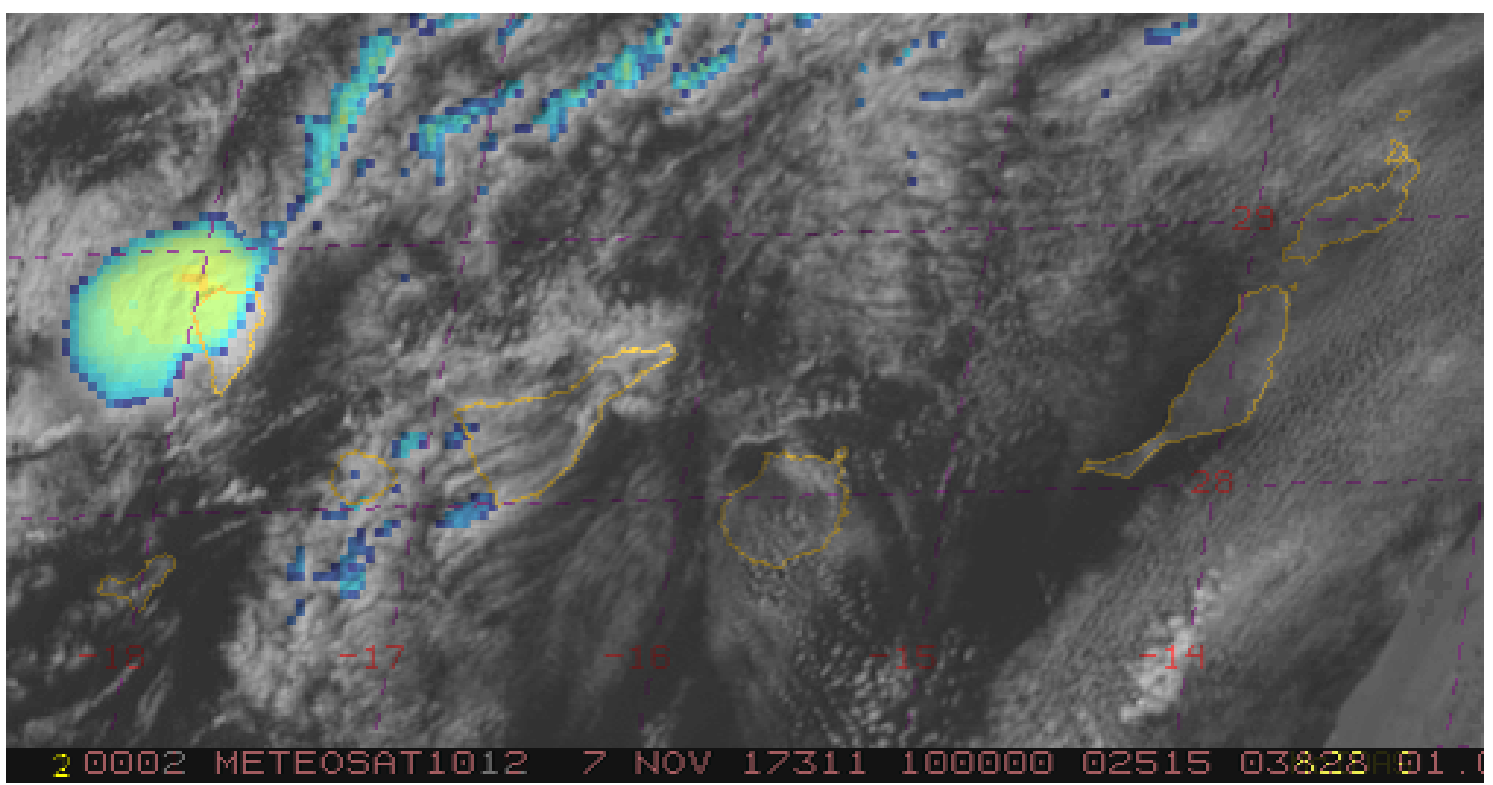

Figura 13. Imagen satélite que muestra el producto sándwich, de las 10 UTC del 7 de noviembre de 2017. 


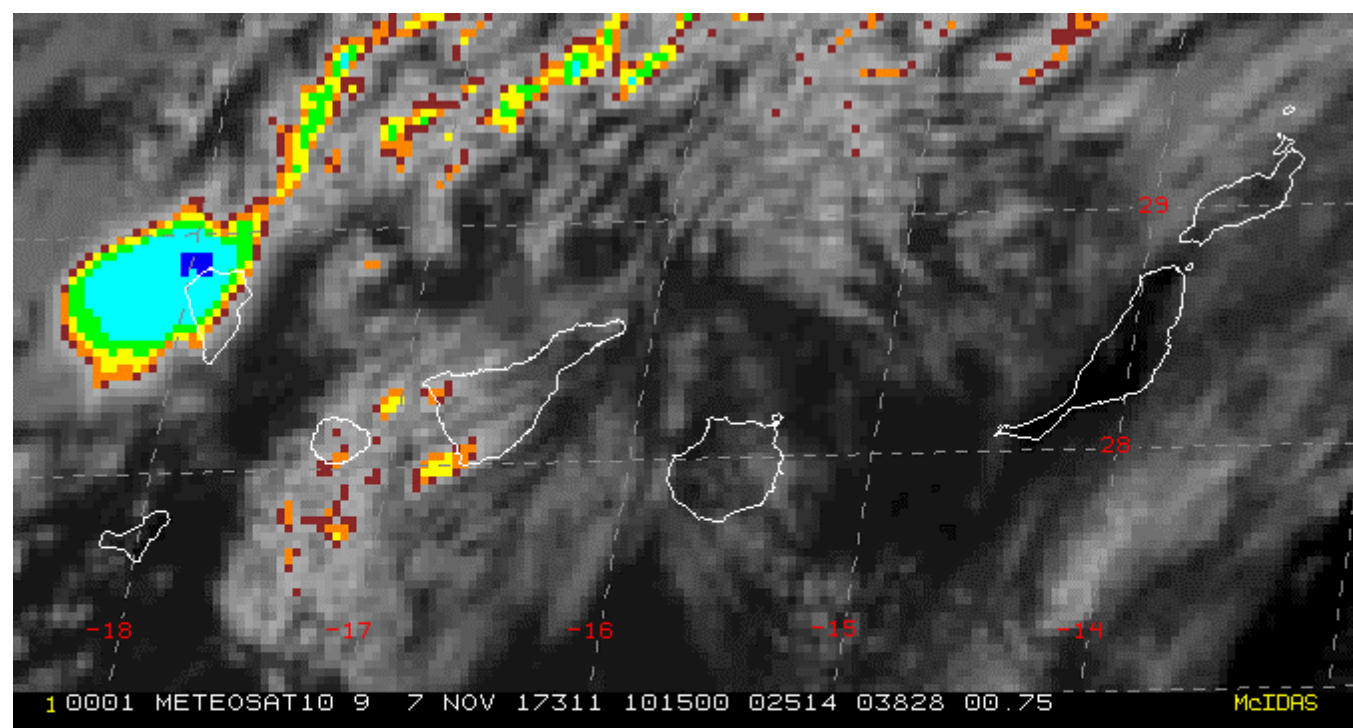

IR10,8 con realce PREVIMET

300

280

260

$\begin{array}{lllll}240 & 234 & 226 & 218 & 210\end{array}$

(K)

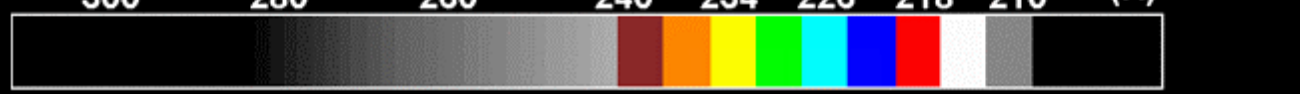

Figura 14. Imagen satélite que muestra el canal IR10.8 con realce PREVIMET, de las 10:15 UTC del 7 de noviembre de 2017.

\section{CONCLUSIONES}

Es evidente que nos enfrentábamos a una situación impredecible al tratarse de un fenómeno de convección muy local, lo que siempre supone un reto para la predicción, máxime en Canarias (QUINTERO y SUÁREZ, 2016).

Hemos constatado que a pesar de las características y de la mejor resolución espaciotemporal del modelo Harmonie-Arome, este no ha reflejado una mejora en la predicción de la precipitación respecto a la ofrecida por el modelo determinista HRES-IFS.

Por otro lado sería ideal encontrar un criterio objetivo para poner en práctica cuando nos enfrentamos a este tipo de situaciones, ya que es necesario ser capaces de resolver algunas cuestiones:

- Al tratarse de eventos tan locales e impredecibles, ¿es más acertado tratarlos como puntos singulares o es preferible emitir avisos para zonas más extensas?

- Desde el punto de vista del usuario (protección civil), ¿es eficiente para su toma de decisiones recibir información como llamadas telefónicas sin la necesidad de tener en vigor avisos por FMA (fenómenos meteorológicos adversos)? O, por el contrario, ¿esta información no es útil sin estar sustentada en avisos por FMA para sus tareas de planificación frente a potenciales peligros debidos a condiciones meteorológicas adversas?

- En cuanto a las tareas de vigilancia, está claro que el aumento de la cobertura de datos de observación provenientes de estaciones meteorológicas externas a la red de AEMET, es una cuestión positiva, pero ¿debe el predictor fiarse de cualquier estación sin conocer el mantenimiento ni los estándares de calidad que ha pasado dicha estación? Quizás sería interesante realizar un filtrado de estaciones fiables y establecer un criterio objetivo sobre esta cuestión, ya que si no se podrían manifestar opiniones subjetivas y sesgadas de cuando es «favorable» $\mathrm{o}$ «no favorable» un dato en cuestión. 


\section{AGRADECIMIENTOS}

El autor principal de este artículo quiere agradecer la implicación de los predictores del GPV de Canarias Cristo y Javier de Luis, ya que han dedicado parte de su tiempo libre al análisis, elaboración y revisión de este trabajo.

\section{REFERENCIAS}

Holton, J. R., 2004. An introduction to dynamic meteorology (fourth edition). Academic Press.

LehKonen, A., 2013. Synoptic Meteorology. Eumentrain.

MoRÁn, F., 1984. Apuntes de termodinámica de la atmósfera. INM.

Quintero, D. y SuÁrez, D., 2016. Estudio de la situación de precipitaciones intensas de los días 22 y 23 de octubre de 2015 en Canarias. Nota técnica 22. AEMET. 